\title{
Load Frequency Control of Power Systems Based on ADRC
}

\author{
Qian Liu*, Meikui He, Zhen Fang, Ting Gui, Changqing Dong, Jing An \\ Wuhan second Marine Design and Research Institute, Wuhan Hubei, 430064, China
}

\begin{abstract}
Load frequency stability is an important power quality index in power system, any sudden load disturbance will cause the system load frequency deviation, and as the power system becomes more complex, the difficulty of control is also increasing, it is necessary to find a more appropriate control method. Considering that the load frequency control system model has the characteristics of multi-variable and strong coupling, combines the superior anti-interference and anti-coupling ability of the active disturbance rejection control, a novel scheme for power system load frequency control was presented based on the principle of active disturbance rejection control and effective open-loop transfer function. The simulation results show that the proposed method is simple tuning and strong decoupling ability, and provides a successful control of load frequency.
\end{abstract}

\section{Introduction}

Load Fequency Control (LFC) is very important. And the load of power system is changing at any time, in order to ensure the stability of power system frequency, it is necessary to design a suitable load frequency controller.

Active disturbance rejection control (ADRC) was first proposed by Han in $1990 \mathrm{~s}^{[1]}$. The central idea of ADRC is that both the internal dynamics and the external disturbances such as the interactions among different loops and various uncertainties can be viewed as a general disturbance, and it can be estimated and compensated in real time by using an extended state observer(ESO) ${ }^{[2]}$.ADRC has obtained good control effect in the simulation of a large number of control systems such as power system ${ }^{[3]}$ and aircraft ${ }^{[4]}$, showing a great application prospect. For multivariable systems with weak coupling or approximate decoupling, the paper ${ }^{[5]}$ designs the ADRC controller for each loop based on the diagonal model. Because the coupling between loops is not considered in this method, the parameters of the decentralized controller need to be adjusted repeatedly to obtain good control effect, and the design process is tedious and the workload is large.

$\mathrm{Vu}$ and Lee ${ }^{[6]}$ first proposed the concept of effective open-loop transfer function (EOTF) to design of multiloop PI/PID controllers. EOTF is an approximated model, can't completely describe the cross coupling between loops, so the decoupling performance of multi-loop $\mathrm{PI} / \mathrm{PID}$ controllers based on EOTF method is possible to be improved.

LFC system as a typical model of multivariable coupling system, in order to solve the above problems, a novel method of ADRC controller design is introduced. This method is based on ADRC excellent tracking ability to resist disturbance, combined with EOTF concept to guide the design of decentralized ADRC controller, coupling information can be included, and can make use of ESO estimate ability to fully offset the additional error of equivalent process. Therefore, the design process can be greatly simplified. The proposed method is applied to the LFC model, and the simulation results verify the effectiveness of the proposed method.

\section{LFC system model}

\subsection{LFC for Single-Area Power Systems}

We first consider the case of a single generator supplying power to a single service area. The linear model can be shown in Figure1.

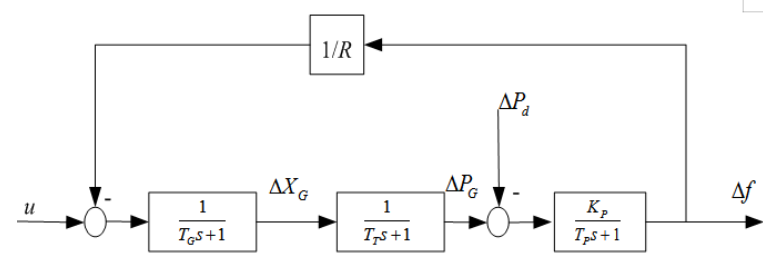

Fig. 1. Single-Area Power System

The linear model is composed of three parts, and the linear model of each part is shown as follows:

The governor dynamics: $G_{g}(s)=\frac{1}{T_{G} s+1}$

The turbine dynamics: ${ }_{G_{t}}(s)=\frac{1}{T_{T} s+1}$

The power system dynamics: $G_{P}=\frac{K_{P}}{T_{P} s+1}$

Where the $T_{P}, T_{T}, T_{G}$ are the time constants. $1 / \mathrm{R}$ is the unit droop characteristics, $K_{P}$ is the power generation

\footnotetext{
*Corresponding author: liuqian_bj@163.com
} 
system gain, $\Delta X_{G}$ is the corresponding governor value change (p.u.mw), $\Delta P_{G}$ is the corresponding generator output change (p.u.mw), $\Delta P_{d}$ is the load disturbance (p.u.mw), $\Delta f$ is the corresponding frequency deviation.

\subsection{LFC for Multi-Area Power Systems}

In fact, power systems in various regions are connected to other regional systems through tie lines. With the expansion of power grids, the number of nodes in interconnected power systems is also increasing, and they are developing towards the joint operation of large power grids. Therefore, it is of more practical significance to study the frequency control of multiregion interconnected power systems. Its structure is shown in figure 2.

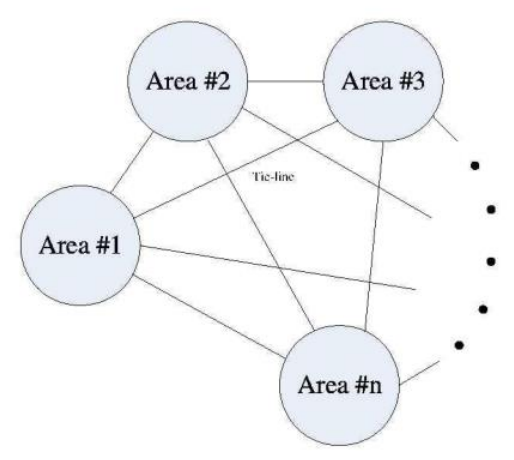

Fig. 2. Multi-Area Interconnection Power System

And each area has the structure shown in figure 3.

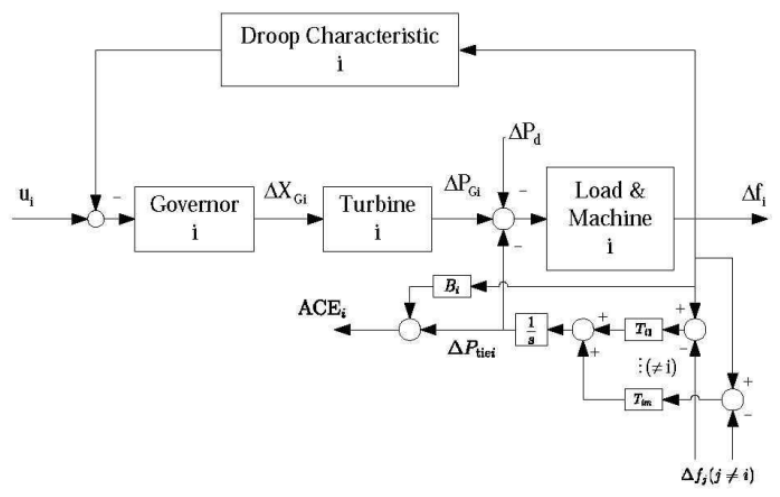

Fig. 3. Block diagram of Area $i$

The multi-area power system load frequency control is not only to ensuring the frequency stability of each region, but also the tie line power flows must return to their expected values. Therefore, a compound variable, the area control error (ACE), is used to represent the system output:

$$
A C E_{i}=\Delta P_{\text {tiei }}+B_{i} \Delta f_{i}
$$

Where, $\Delta P_{\text {tiei }}$ is the tie line power flows.

$$
\Delta P_{\text {tiei }}=\frac{1}{S} \sum_{j}^{n} T_{i j}\left[\Delta f_{i}-\Delta f_{j}\right],(i \neq j)
$$

Where $B_{i}$ is the frequency bias setting, $\Delta f_{i}$ is the frequency deviation. $T_{i j}$ shows the interconnection constant between area $i$ and area $j$. According to the figure, the control law of area $i$ can be expressed as follows:

$$
u_{i}=-K_{i}(\mathrm{~s}) \mathrm{ACE}_{i}
$$

Above all, the load frequency control problem of power system is mainly a problem of multivariable coupling disturbance suppression. Although multivariable control technology has been greatly developed in recent years, decentralized control structure is mostly used for load frequency control, but the general controller design process is relatively complicated.

\section{ADRC controller Design based on EOTF}

\subsection{Basic principle of ADRC}

We consider a 2-order linear ADRC, the control block diagram is showed in Fig.2.

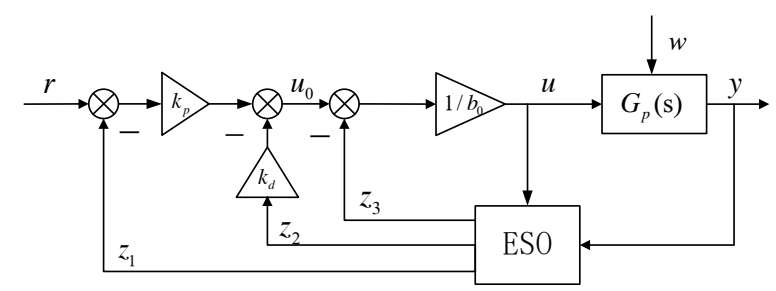

Fig.4. Block diagram of second-order linear ADRC

where $r$ is set point and $y$ is output, $G_{p}$ is the plant, $k_{p}, k_{d}$ and $b_{o}$ is the controller parameters to be tuned. The ESO can estimate and compensate the external disturbances $(w)$ and system uncertainties in real time, $z_{1}, z_{2}$ and $z_{3}$ is the output of ESO. The expression of ESO is

$$
\left\{\begin{array}{l}
\dot{z}_{1}=z_{2}-\beta_{1}\left(z_{1}-y\right) \\
\dot{z}_{2}=z_{3}-\beta_{2}\left(z_{1}-y\right)+b_{0} u \\
\dot{z}_{3}=\beta_{3}\left(z_{1}-y\right)
\end{array}\right.
$$

where $\beta_{1}, \beta_{2}, \beta_{3}$ are ESO parameters to be tuned.

Suppose $G_{p}$ can be approximated to a second order plant,

$$
\ddot{y}=g(t, y, \dot{y}, \cdots, w)+b u
$$

where $\mathrm{b}$ is a constant, $g(t, y, \dot{y}, \cdots, w)$ represents the nonlinear time-varying dynamics of the plant that is unknown. Define an extended state,

$$
f=g+\left(b-b_{0}\right) u
$$

With a well-tuned ESO as(9), the outputs of $z_{1}, z_{2}, z_{3}$ can closely track $y, \dot{y}, f$, respectively. According to Fig.4, the control law is given by

$$
\begin{gathered}
u_{0}=k_{p}\left(r-z_{1}\right)-k_{d} z_{2} \\
u=\left(u_{0}-z_{3}\right) / b_{0}
\end{gathered}
$$
plant

The original plant will be simplified into an integral

$$
\ddot{y}=f+u_{0}-z_{3} \approx u_{0}
$$

\subsection{The EOTF Design}

As shown in figure 5, the dimension process, the loop $i$ is open and the other loops are closed. 


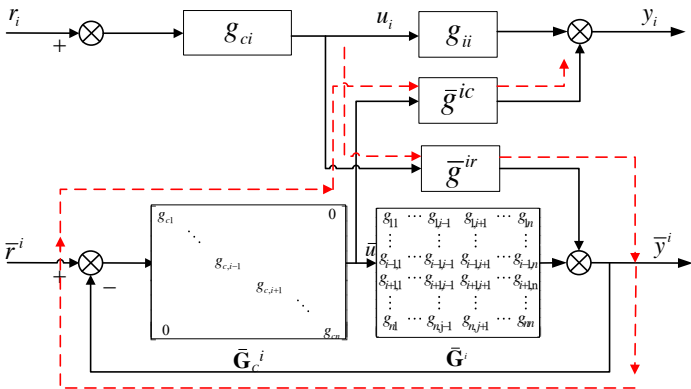

Fig. 5 Block diagram for the concept of the EOTF in a $n \times n$ multi-loop system

Where, $\overline{\mathbf{r}}^{i}, \overline{\mathbf{u}}^{i}, \overline{\mathbf{y}}^{i}$ are the set point, manipulated, and output variable vectors, where $r_{i}, u_{i}, \mathrm{y}_{i}$ are dropped from $\mathbf{r}, \mathbf{u}, \mathbf{y}$.

With $\overline{\mathbf{r}}^{i}=0, \overline{\mathbf{u}}^{i}$ is obtained as

$$
\overline{\mathbf{u}}^{i}=-\tilde{\mathbf{G}}_{c}^{i} \overline{\mathbf{y}}^{i}=-\tilde{\mathbf{G}}_{c}^{i}\left(\bar{g}^{i c} u_{i}+\overline{\mathbf{G}}^{i} \overline{\mathbf{u}}^{i}\right)
$$

Assuming that $\overline{\mathbf{G}}^{i} \tilde{\mathbf{G}}_{c}^{i}\left(\mathbf{I}+\overline{\mathbf{G}}^{i} \tilde{\mathbf{G}}_{c}^{i}\right)^{-1}=\mathbf{I}$ as a perfect control, then the EOTF of the $i$ th loop is

$$
g_{i i}^{e f f}=g_{i i}-\bar{g}^{i r}\left(\overline{\mathbf{G}}^{i}\right)^{-1} \bar{g}^{i c}
$$

Therefore, combined with the definition of dynamic relative gain matrix (DRGA), $\Lambda_{i i}=\left[\mathrm{G} \otimes\left(\mathrm{G}^{-1}\right)^{T}\right]_{i i}$, so the EOTF can be expressed as follows,

$$
g_{i i}^{e f f}=\frac{g_{i i}}{\Lambda_{i i}}
$$

The $i$ th loop that $u_{i}$ to $y_{i}$ (as Fig.5) can be approximated to the structure as shown in Fig.6. So the original multi-loop system can be equivalent to $n$ such single loops ${ }^{[7]}$.

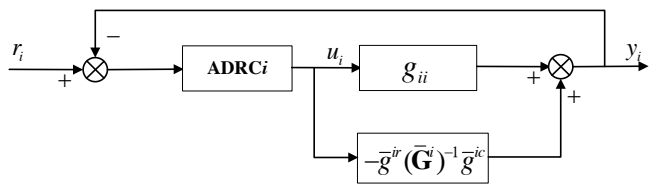

Fig. 6. Approximation of structure in Fig. 5

\section{Simulation study}

In this paper, the classical two-area and three-area interconnected power systems are selected as simulation objects, and the model errors in the process of model simplification and order reduction are considered as disturbances by using the EOTF solution introduced in this paper. ADRC controllers are designed to achieve better control performance by using ADRC error estimation and compensation capability. Paper [8] proposed a PID control strategy based on internal module design, and achieved a good control effect. In this paper, the proposed scheme is used for simulation analysis and compared with the PID scheme.

\subsection{Two-Area Interconnected Power System}

Consider the two-area interconnection power system model shown in the figure below, the units in the two areas have the same structure.

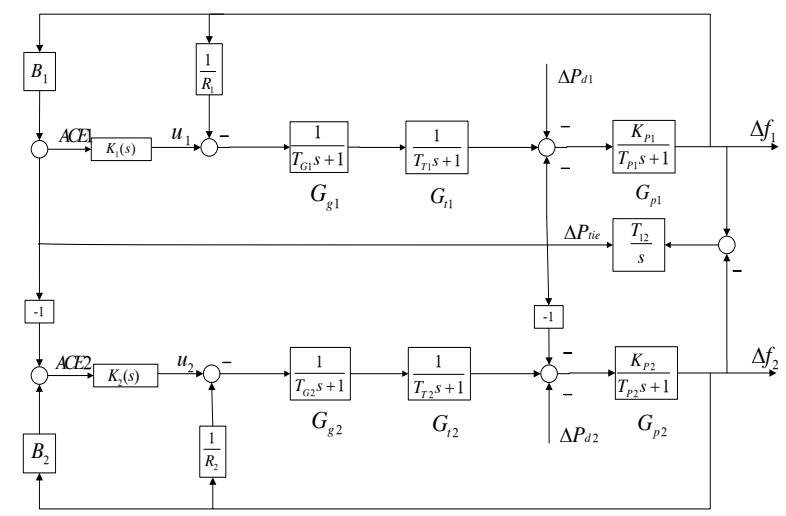

Fig. 7 Two-Area Interconnected Power System

Model parameters as follows:

$T_{G 1}=T_{G 2}=0.08, T_{T 1}=T_{T 2}=0.3, T_{P 1}=T_{P 2}=20$,

$K_{P 1}=K_{P 2}=120, R_{1}=R_{2}=2.4$,

$T_{12}=0.545, B_{1}=B_{2}=0.425$

The frequency deviations of area 1and area 2 are $\Delta f_{1}, \Delta f_{2}$

$$
\Delta P_{\text {tie }}=\frac{T_{12}}{S}\left(\Delta f_{1}-\Delta f_{2}\right)
$$

The area control error of the two areas are $\mathrm{ACE}_{1}$, $\mathrm{ACE}_{2}$,

$$
\begin{aligned}
& A C E_{1}=\Delta P_{\text {tie }}+B_{1} \Delta f_{1} \\
& A C E_{2}=-\Delta P_{\text {tie }}+B_{2} \Delta f_{2}
\end{aligned}
$$

From the Fig.7, the model can be defined as follows

$$
\left[\begin{array}{c}
A C E_{1} \\
A C E_{2}
\end{array}\right]=\left[\begin{array}{cc}
B_{1}+\frac{T_{12}}{s} & -\frac{T_{12}}{s} \\
-\frac{T_{12}}{s} & B_{2}+\frac{T_{12}}{s}
\end{array}\right]\left[\begin{array}{l}
\Delta f_{1} \\
\Delta f_{2}
\end{array}\right]
$$

$$
\left[\begin{array}{l}
\Delta f_{1} \\
\Delta f_{2}
\end{array}\right]=\left[\begin{array}{cc}
1+\frac{G_{p 1} G_{t 1} G_{g 1}}{R_{1}}+\frac{G_{p 1} T_{12}}{s} & -\frac{G_{p 1} T_{12}}{s} \\
-\frac{G_{p 2} T_{12}}{s} & 1+\frac{G_{p 2} G_{t 2} G_{g 2}}{R_{2}}+\frac{G_{p 2} T_{12}}{s}
\end{array}\right]^{-1}\left[\begin{array}{cc}
G_{p 1} G_{t 1} G_{g 1} & 0 \\
0 & G_{p 2} G_{t 2} G_{g 2}
\end{array}\right]\left[\begin{array}{l}
u_{1} \\
u_{2}
\end{array}\right]
$$

In order to fully utilize the coupling information of the model, the interconnections between the two areas are not ignored here. Instead, the concept of EOTF was introduced. The equivalent object after the model is reduced order is given directly here

$$
\begin{aligned}
A C E_{1}^{r_{-} \text {eff }} & =A C E_{2}^{r_{-} \text {eff }} \\
& =\frac{0.3897 s+1}{0.003658 s^{4}+0.06297 s^{3}+0.2425 s^{2}+0.7795 s+1}
\end{aligned}
$$

According to the above two equivalent model, the ADRC strategy is designed for the two-area load frequency control system and compared with the PID scheme. The ADRC controller was designed for equation (20), and then applied to the original two-area model.

Assuming that at $t=1 \mathrm{~s}$, area 1 is disturbed by load of 0.01 p.u. When $\mathrm{t}=15 \mathrm{~s}$, area 2 is disturbed by load of 0.01p.u.The PID scheme and the ADRC control scheme 
proposed in this paper are adopted respectively. The variation curves of frequency deviation and tie line exchange power in each region are as follows.
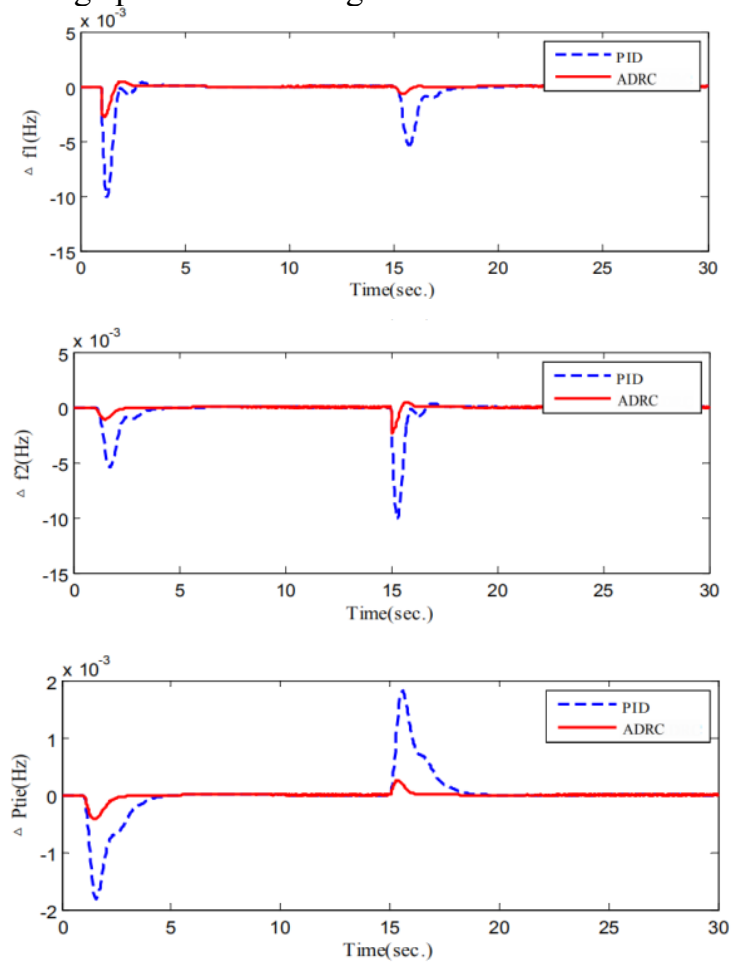

Fig. 8. The Response of the Two-Area LFC

The figure shows that the ADRC controller interference resistance and decoupling ability is better than PID controller. When each area is disturbed by the same load, the PID control scheme has been able to control the frequency deviation in a small range, but ADRC with its superior disturbance rejection ability can ensure that the system is subject to load disturbance, the frequency deviation is smaller, and can recover to balance more quickly.

\subsection{Three-Aera Interconnected Power System}

In order to verify the effectiveness of the proposed method and its adaptability to objects of different orders, a three-area interconnected power system model is selected. The transfer function as follows:

$$
G_{g i}(s)=\frac{1}{T_{G i} s+1}, G_{t i}(s)=\frac{1}{T_{T i} s+1}, G_{P i}=\frac{K_{P i}}{T_{P i} s+1},(i=1,2,3)
$$

The model parameters of different areas are as follows:

$T_{G 1}=0.08, T_{T 1}=0.3, T_{P 1}=20, K_{P 1}=120$,

$T_{G 2}=0.072, T_{T 2}=0.33, T_{P 1}=25, K_{P 1}=112.5$,

$T_{G 1}=0.07, T_{T 1}=0.35, T_{P 1}=20, K_{P 1}=115$,

$T=0.5, B_{1}=B_{2}=B_{3}=0.425$

The simplest equivalent model is obtained as:

$$
\begin{aligned}
A C E_{1}^{r-\text { eff }} & =\frac{0.7135 s+0.9799}{0.009294 s^{4}+0.07728 s^{3}+0.3589 s^{2}+1.126 s+1} \\
A C E_{2}^{r-\text { eff }} & =\frac{0.6431 s+1.017}{0.01241 s^{4}+0.1073 s^{3}+0.4114 s^{2}+1.086 s+1} \\
A C E_{3}^{r-\text { eff }} & =\frac{1.377 s+0.9926}{0.02196 s^{4}+0.1375 s^{3}+0.6728 s^{2}+1.815 s+1}
\end{aligned}
$$

Assuming that at $t=1 \mathrm{~s}$, area 1 is disturbed by load of $0.01 \mathrm{p} . \mathrm{u}, \mathrm{t}=10 \mathrm{~s}$, area 2 is disturbed by load of $0.01 \mathrm{p} . \mathrm{u}$. $\mathrm{t}=20 \mathrm{~s}$, area 3 is disturbed by load of $0.01 \mathrm{p}$.u. Also compared with the IMC- PID control scheme, the dynamic response of the system is shown below.
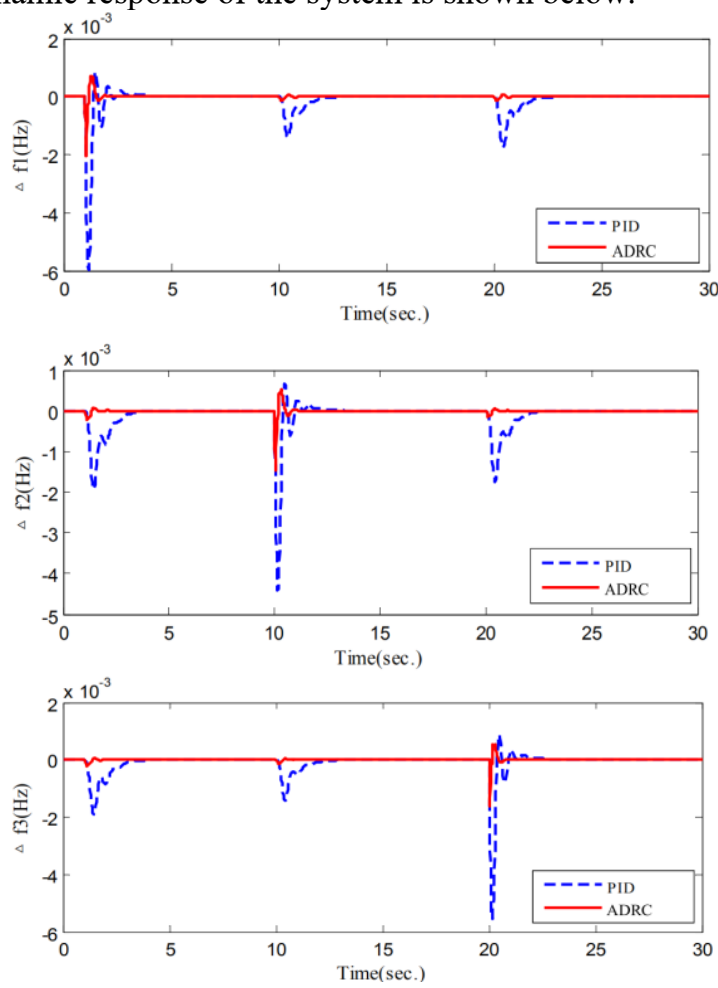

Fig. 9. The Frequency deviation of the Three-Area LFC
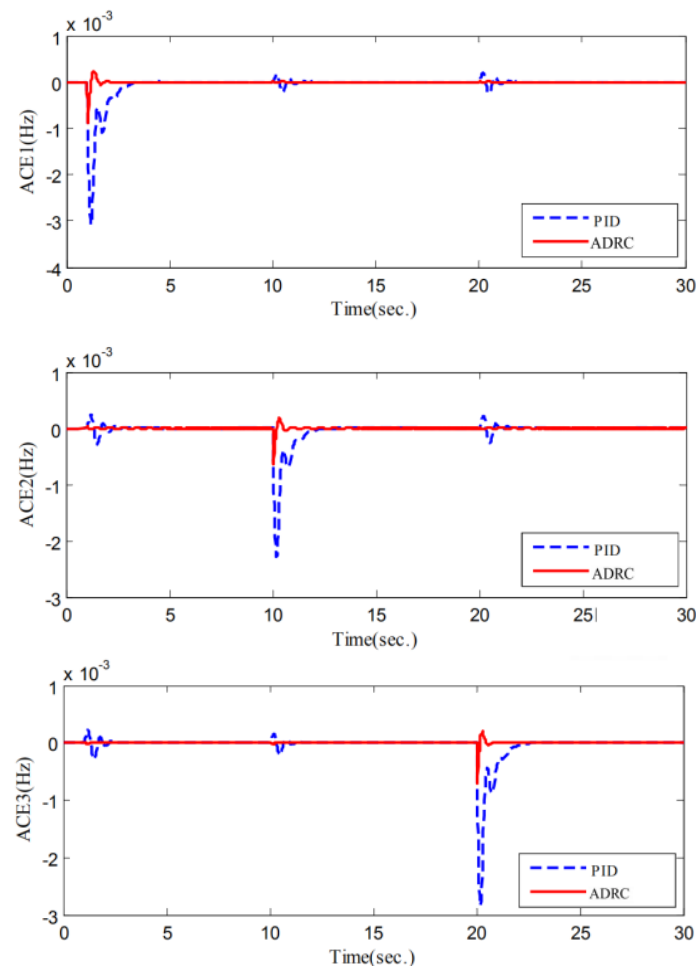

Fig. 10. The ACE of the Three-Area LFC

As can be seen from the load frequency response curve, the method proposed in this paper can also achieve good control effect on three-region objects. 
When the system is disturbed by load, ADRC controller can better control the frequency deviation in a smaller range, and the design process is simple, and the control effect is improved a lot on the basis of PID.

\section{Conclusion}

For the strong coupling characteristics of multivariable load frequency control system model, an ADRC control strategy based on EOTF is proposed. For the problem of multi-area load frequency control system, when different areas set different controllers, because of the coupling effect, the parameters need to be adjusted repeatedly. This paper successfully introduced the concept of EOTF, firstly, the multi-area model is reduced to a single equivalent object under the condition of making full use of the coupling information, and then the controller is designed for the equivalent object. Simulation results show that the proposed method has good control effect and performance, and the design process is simple.

\section{References}

1. J. Han From PID to active disturbance rejection control. Industrial Electronics, IEEE transactions on, 56(3): 900-906, 2009.

2. J. Han, Active Disturbance Rejection Control Technique- the technique for estimating and compensating the uncertainties, Beijing,2009

3. W Tan, H Zhou, CF Fu. Linner Active Diturbence Rejection control for Load Frequency Control of Power System[J]. Control Theory\& Applications, 30(12): 1580-1588,2013.

4. WC Xue, CD Huang, Y Huang. Design Methods for the Integrated Guidance and Control System[J]. Control Theory\& Applications, 30(12): 1511$1521,2013$.

5. LL TIAN, DH. LI and CE HUNG. Decentralized Controller Design Based on 3-order Active Disturbance Rejection Control, Proceedings of the 10th World Congress on Intelligent Control and Automation, 2746-2751,2012.

6. $\mathrm{Vu} \mathrm{T} \mathrm{N} \mathrm{L}$, Lee M. Independent design of multi-loop PI/PID controllers for interacting multivariable processes. Journal of Process control, 20(8): 922933, 2010.

7. Q Liu, DH Li, E Tan. Design of Multi-loop ADRC Controllers Based on the Effective Open-loop Transfer Function Method[C].2014CCC:3649-3652.

8. W Tan. Tuning of PID load frequency controller for power systems[J]. Energy Conversion and Management, 50(6): 1465-1472,2009. 